\title{
ALTERAÇÕES NOS ATRIBUTOS QUÍMICOS DE UM LATOSSOLO PELO MANEJO DE PLANTAS INVASORAS EM CAFEEIROS ${ }^{(1)}$
}

\author{
Cezar Francisco Araujo-Junior ${ }^{(2)}$,Paulo Tácito Gontijo Guimarães ${ }^{(3)}$, \\ Moacir de Souza Dias Junior ${ }^{(4)}$, Elifas Nunes Alcântara ${ }^{(3)}$ \& Aretusa \\ Daniela Resende Mendes ${ }^{(5)}$
}

\begin{abstract}
RESUMO
O controle de plantas invasoras é uma das práticas de manejo mais intensivas na condução de lavouras cafeeiras, o qual provoca alterações nos atributos químicos do solo. Diante disso, os objetivos deste estudo foram: avaliar os efeitos dos diferentes sistemas de manejo de plantas invasoras em uma lavoura cafeeira nos atributos químicos de um Latossolo Vermelho distroférrico em relação ao solo sob mata nativa (MATA); e verificar a relação entre o teor de C orgânico do solo (COS) e a capacidade de troca de cátions efetiva (CTC a pH natural) e a capacidade de troca de cátions a pH 7 (CTC a pH 7). O estudo foi realizado na fazenda experimental da Empresa de Pesquisa Agropecuária de Minas Gerais (EPAMIG), localizada no município de São Sebastião do Paraíso, Minas Gerais. A área experimental foi plantada com cafeeiros da cultivar Paraíso, e o experimento foi instalado em blocos casualizados, com sete manejos de plantas invasoras e três repetições. Os manejos de plantas invasoras avaliados foram: sem capina (SCAP); capina manual (CAPM); herbicida de pós-emergência (HPOS); roçadora (ROÇA); enxada rotativa (ENRT); grade (GRAD); e herbicida de pré-emergência (HPRE). Cada manejo de plantas invasoras nas entrelinhas dos cafeeiros vem sendo realizado há 30 anos em três ruas, com $36 \mathrm{~m}$ de comprimento cada. As amostras de solo foram coletadas no centro das entrelinhas dos cafeeiros em dezembro de 2007, com cinco amostras
\end{abstract}

(1) Trabalho apresentado no VI Simpósio de Pesquisa dos Cafés do Brasil: Inovação Científica, Competitividade e Mudanças Climáticas, 2009. Vitória (ES). Recebido para publicação em 26 de novembro de 2010 e aprovado em 17 de agosto de 2011.

(2) Eng.-Agr., MSc., Dr. Pesquisador da Área de Solos do Instituto Agronômico do Paraná - IAPAR. Caixa Postal 48, CEP 86001970 Londrina (PR). E-mail: cezar_araujo@iapar.br; cfaj@bol.com.br

(3) Engenheiro-Agrônomo, Dr. Pesquisador do Centro Tecnológico do Sul de Minas, Empresa de Pesquisa Agropecuária de Minas Gerais (CTSM/EPAMIG). Campus da UFLA. Caixa Postal 176, CEP 37200-000 Lavras (MG). E-mails: paulotgg@epamig.ufla.br; elifas@epamig.ufla.br

(4) Engenheiro Agrícola, PhD in Crop and Soil Science, Professor Associado do Departamento de Ciência do Solo, UFLA. Bolsista do CNPq e da FAPEMIG - Pesquisador Mineiro. E-mail: msouzadj@dcs.ufla.br

(5) Eng. Agrônoma, MSc., Dra., Instituto de Ciências Agrárias, Universidade Federal de Minas Gerais - UFMG. Av. Universitária 1000, Bairro Universitário, CEP 39404-006 Montes Claros (MG). Bolsista do Programa Nacional de Pós Doutorado - PNPD/ CAPES. E-mail: are.dani@hotmail.com 
simples por parcela, que perfizeram uma amostra composta em três profundidades $(0-3,10-13$ e $25-28 \mathrm{~cm})$. As seguintes análises químicas foram realizadas nas amostras de solo: pH em água, cátions trocáveis (Ca, Mg, K e $\mathrm{Al}$ ), $\mathrm{C}$ orgânico (COS), capacidade de troca de cátions efetiva ou a pH natural (CTC efetiva) e capacidade de troca de cátions a pH 7. Os manejos apresentaram características contrastantes, variando desde métodos manuais até capinas químicas e mecânicas. Os resultados permitiram observar que a manutenção das plantas invasoras nas entrelinhas dos cafeeiros, adotada no manejo sem capina, contribuiu positivamente para as alterações dos atributos químicos (Ca trocável, CTC efetiva e CTC a pH 7,0) nas três profundidades estudadas; além disso, elevou o teor de $\mathrm{C}$ orgânico total na profundidade de 0-3 cm e pode contribuir para aumento e manutenção dos estoques de C em lavouras cafeeiras. Assim, o manejo SCAP nas entrelinhas dos cafeeiros pode ser adotado para a melhoria e manutenção dos atributos químicos em lavouras cafeeiras. Por outro lado, a utilização constante e por longo prazo (30 anos) do manejo HPRE reduziu os valores de pH nas profundidades de 10-13 e 25-28 cm e do Ca trocável, Mg trocável e CTC efetiva nas três profundidades estudadas, em relação aos demais manejos de plantas invasoras. Os valores de CTC efetiva do Latossolo Vermelho distroférrico apresentaram relação com o teor de $\mathrm{C}$ orgânico em 59, 60 e $47 \%$ dos casos e de CTC a pH 7 em 65, 55 e $46 \%$, nas profundidades de 0-3, 10-13 e 25-28 cm.

Termos de indexação: controle de plantas daninhas, carbono orgânico total, capacidade de troca de cátions, cargas do solo, $\mathrm{pH}$, eletroquímica de solo.

\section{SUMMARY: CHANGES IN CHEMICAL PROPERTIES OF A LATOSOL BY WEED MANAGEMENT IN COFFEE PLANTIONS}

Weed control is one of the most intensive management practices in coffee plantations resulting in changes in soil chemical properties. The objectives of this study were: a) to assess the effects of weed management in a coffee plantation on the chemical properties of a Dystroferric Red Latosol (Oxisol) compared to soil under native forest; $b$ ) to verify the relationship between soil organic carbon content (COS) and the effective cation exchange capacity (effective CEC) and the cation exchange capacity at $p H$ 7. This study was carried out on an experimental farm of the Agricultural Research Company of Minas Gerais (EPAMIG) at the São Sebastião do Paraíso County, of Minas Gerais State. In the experimental area, coffee was planted and the experiment was installed in randomized blocks with three replications. The following weed plant managements were evaluated: without weed control (SCAP); hand weeding (CAPM); post-emergence herbicide (HPOS); mechanized mower (ROÇA); rotary tiller (ENRT); coffee tandem disc harrow (GRAD) and pre-emergence herbicide (HPRE). Each one of these weed control systems had been performed in the preceding 30 years in three inter-rows (length $36 \mathrm{~m}$ ). Five soil samples were collected in three layers $(0-3,10-13$ and $25-28 \mathrm{~cm})$ per plot between coffee rows, in December 2007. The following soil chemical analyses were performed: $p H$-water, exchangeable cations $(\mathrm{Ca}, \mathrm{Mg}, \mathrm{K}$ and $\mathrm{Al}$ ), cation exchange capacity at natural $\mathrm{pH}$ (effective CEC) and cation exchange capacity at pH 7.0 (CEC potential. The results showed that the maintenance of weed plants between coffee rows in the treatment no weed control (SCAP) had positive effects on the chemical properties (exchangeable Ca, effective CEC and CEC at pH 7.0 in the three layers). Furthermore, the total soil organic carbon content at $0-3 \mathrm{~cm}$ depth increased and may contribute to the increase and maintenance of carbon stocks in coffee plantations. Thus, no weed control (SCAP) between coffee rows can be adopted for the improvement and maintenance of the chemical properties in coffee plantations. On the other hand, the constant long term-use (30 years) with HPRE management decreases the $\mathrm{pH}$-water values in the layers 10-13 and 25-28 cm and the exchangeable $\mathrm{Ca}, \mathrm{Mg}$ and effective CEC in the three layers studied in relation to other weed plant managements. The effective CEC of the soil was related with organic carbon content in 59, 60 and $47 \%$ of the cases and CEC at pH 7 in 65, 55 and $46 \%$ in the layers $0-3,10-13$ and $25-28 \mathrm{~cm}$.

Index terms: weed control; total organic carbon; cation exchange capacity; soil charge; $p H$; soil electrochemistry. 


\section{INTRODUÇÃO}

O controle de plantas invasoras é uma das práticas de manejo mais intensivas na condução de lavouras cafeeiras, o qual provoca alterações na densidade e diversidade dessas plantas, alterando, consequentemente, a produção de biomassa vegetal depositada na superfície do solo e, possivelmente, a dinâmica do C orgânico e os atributos químicos do solo.

A densidade e diversidade de plantas invasoras que ocorrem na lavoura cafeeira podem estar relacionadas com o tipo de manejo (manual, mecânico ou químico) adotado na área (Alcântara \& Ferreira, 2000b), bem como promover alterações na quantidade e na qualidade da matéria orgânica, alterando a reação do solo, a solubilidade de Al e a adsorção de $\mathrm{Ca}, \mathrm{Mg}$ e K (Pavan et al., 1995). Além do tipo de manejo adotado, o número médio de operações anuais para o controle satisfatório das plantas invasoras pode alterar a produção da lavoura cafeeira (Alcântara \& Ferreira, 2000a; Alcântara \& Ferreira, 2009).

Os Latossolos são solos altamente intemperizados, ácidos e caracterizados pela remoção de $\mathrm{SiO}_{2}$ e acúmulo relativo de $\mathrm{Al}\left(\mathrm{Al}_{2} \mathrm{O}_{3}\right)$ na sua fase mineral. São solos constituídos predominantemente pelos minerais caulinita (CTC $\left.=0-1 \mathrm{cmol}_{\mathrm{c}} \mathrm{kg}^{-1}\right)$, óxidos de ferro (CTC $=2-4 \mathrm{cmol}_{\mathrm{c}} \mathrm{kg}^{-1}$ hematita e goethita) e hidróxidos de $\mathrm{Al}$ (CTC $=2-4 \mathrm{cmol}_{\mathrm{c}} \mathrm{kg}^{-1}$ gibbsita) (Meurer et al., 2006). A CTC medida para a caulinita pode ser variável $\left(\mathrm{CTC}=2-15 \mathrm{cmol}_{\mathrm{c}} \mathrm{kg}^{-1}\right)$, dependendo do grau de impureza na argila e do valor do $\mathrm{pH}$ no qual essa propriedade foi medida (Sparks, 1995). Entretanto, se poucas impurezas são verificadas, a CTC medida pode ser menor que $2 \mathrm{cmol}_{\mathrm{c}} \mathrm{kg}^{-1}$ (Sparks, 1995). Esses constituintes contribuem para as cargas do solo, porém conferem-lhe baixa capacidade de troca catiônica, a qual varia com o pH (Agbenin \& Raij, 1999).

No caso dos óxidos de ferro e hidróxidos de $\mathrm{Al}$, dado o ponto de carga zero $\mathrm{PCZ}>8,0$ (valor de $\mathrm{pH}$ em que as cargas negativas igualam-se às cargas positivas $\mathrm{e}$ valores de $\mathrm{pH}$ acima do $\mathrm{PCZ}$ contribuem com cargas negativas e abaixo contribuem com cargas positivas), no $\mathrm{pH}$ dos solos normalmente cultivados $(4,5-6,5)$, esses óxidos estão contribuindo para o predomínio de cargas positivas. Segundo Fontes et al. (2001), os solos altamente intemperizados dos trópicos comportam-se essencialmente como óxidos metálicos e apresentam, predominantemente, cargas dependentes de $\mathrm{pH}$.

A matéria orgânica tem sido apontada como um indicador primário da qualidade do solo, por atuar direta e indiretamente em atributos físicos, químicos e biológicos do solo e sobre as plantas (Alcântara \& Ferreira, 2000a; Doran, 2002; Lal, 2007; Payan et al., 2007; Alcântara et al., 2009). Em razão disso, a escolha correta do sistema de manejo de plantas invasoras a ser empregado em lavouras cafeeiras deve considerar a capacidade de manutenção e, ou, o aumento dos teores de matéria orgânica do solo (Alcântara et al., 2009).
A manutenção da matéria orgânica pode contribuir para a ciclagem de nutrientes e o fluxo de nutrientes para as plantas, além de ser um importante constituinte para a agregação do solo. Além disso, a matéria orgânica acumulada na superfície do solo após cortes sucessivos das plantas invasoras contribui para a formação de novas cargas negativas variáveis no solo, alterando a química da superfície coloidal (Pavan et al., 1995). Esses autores observaram que a matéria orgânica contribuiu de 77 a $81 \%$ para a CTC efetiva e de 56 a $62 \%$ da CTC a pH 7 de um Latossolo cultivado com cafeeiros e submetido a diferentes manejos de plantas invasoras no Paraná. Em amostras subsuperficiais e superficiais de 22 perfis de solos do Estado de São Paulo, Raij (1969) constatou que a fração orgânica dos solos representou de 41 a 74 \% da CTC. Ademais, a CTC da matéria orgânica aumenta com o aumento do $\mathrm{pH}$ (Raij, 1969; Pavan et al., 1985; Agbenin \& Raij, 1999), provavelmente em virtude da dissociação de grupos carboxílicos e fenólicos. Em um estudo na camada de 0-30 cm de sete solos não cultivados do Paraná, Pavan et al. (1985) mostraram que aumentos abrúpticos da CTC com o aumento do $\mathrm{pH}$ foram associados à matéria orgânica do solo, uma vez que, os solos com maiores valores de CTC foram os mais ricos em matéria orgânica.

Em solos tropicais e subtropicais, a CTC da matéria orgânica pode representar um grande percentual da CTC total do solo; a manutenção ou o aumento dos teores de matéria orgânica é fundamental na retenção dos nutrientes e na diminuição da sua lixiviação (Bayer $\&$ Mielniczuk 2008). Nesse sentido, ressalta-se que a manutenção da matéria orgânica em lavouras cafeeiras submetidas a diferentes sistemas de manejo de plantas invasoras é um importante componente para a sustentabilidade dos solos cultivados com cafeeiros.

Os objetivos deste estudo foram: avaliar os efeitos dos diferentes sistemas de manejo de plantas invasoras em uma lavoura cafeeira sobre os atributos químicos de um Latossolo Vermelho distroférrico cultivado com cafeeiros, em relação ao solo sob mata nativa; e verificar a relação entre as variáveis teor de $\mathrm{C}$ orgânico do solo (COS) e capacidade de troca de cátions efetiva (CTC efetiva ou a $\mathrm{pH}$ natural) e a capacidade de troca de cátions a pH 7 (CTC a pH 7,0).

\section{MATERIAL E MÉTODOS}

O estudo foi conduzido na Fazenda Experimental da Empresa de Pesquisa Agropecuária do Estado de Minas Gerais (EPAMIG), localizada no município de São Sebastião do Paraíso, nas mesorregiões Sul e Sudoeste de Minas Gerais, a uma latitude de $20^{\circ} 55^{\prime} 00^{\prime}$ S, longitude $47^{\circ} 07^{\prime} 10^{\prime \prime} \mathrm{W}$ de Greenwich e altitude de $885 \mathrm{~m}$.

O solo da área de estudo foi classificado como Latossolo Vermelho distroférrico (LVdf) (Embrapa, 2006), gibbsítico, $\mathrm{Ki}=0,53$ e $\mathrm{Kr}=0,32$ (Resende \& 
Santana, 1988), argiloso, com $570 \mathrm{~g} \mathrm{~kg}^{-1}$ de argila, $230 \mathrm{~g} \mathrm{~kg}^{-1}$ de silte e $200 \mathrm{~g} \mathrm{~kg}^{-1}$ de areia, na camada de $0-30 \mathrm{~cm}$.

Cafeeiros da cultivar Catuaí Vermelho LCH 20772-5-99 foram plantados no espaçamento de 4 × $1 \mathrm{~m}$, no ano de 1974. O experimento foi instalado no ano de 1977, em delineamento experimental de blocos casualizados, com sete tratamentos e três repetições. Cada condição de manejo foi aplicada em três entrelinhas de $36 \mathrm{~m}$ de comprimento por $2,4 \mathrm{~m}$ de largura. As linhas de plantio foram mantidas sempre limpas, por meio de capina manual ou da aplicação de herbicidas.

Em 26 de dezembro de 2005, devido ao declínio de produção da lavoura implantada no ano de 1974, esta foi substituída por outra da cultivar Paraíso MG H4191. A lavoura anterior foi retirada da área com o auxílio de um subsolador e, posteriormente, fez-se o sulcamento no mesmo local da linha de plantio anterior. Essas operações foram realizadas deixando intacto o efeito dos tratamentos ao longo dos anos nas entrelinhas.

A correção do solo e a fertilização no sulco de plantio foram feitas conforme Comissão de Fertilidade do Solo do Estado de Minas Gerais - CFSEMG (1999), com $5 \mathrm{~L}$ de palha de café por metro linear de sulco de plantio, $200 \mathrm{~g} \mathrm{~m}^{-1}$ de calcário dolomítico, $200 \mathrm{~g} \mathrm{~m}^{-1} \mathrm{de}$ gesso agrícola e $400 \mathrm{~g} \mathrm{~m}^{-1}$ de superfosfato simples. A aplicação de corretivos (calcário e gesso) e fertilizantes foi realizada desde a implantação da lavoura, sobre a projeção dos cafeeiros.

Os manejos de plantas invasoras avaliados foram: a) Sem capina (SCAP): as plantas invasoras foram deixadas em livre crescimento nas entrelinhas dos cafeeiros. Na ocasião da amostragem, as espécies dominantes encontradas na área foram: Marmodica charantia L. (melão-de-são-caetano, Cucurbitaceae); Euphorbia heterophylla L. (leiteira, Euphorbiaceae); Digitaria insularis (L.) Mea ex Ekman (capimamargoso, Poaceae); Panicum maximum Jacq. (capimcolonião, Poaceae); Nicandra physaloides Gaertn. (joáde-capote, Solanaceae); Ipomoea acuminata (corda-deviola, Convolvulaceae); e Amaranthus viridi, (carurude-mancha, Amaranthaceae); b) Capina manual (CAPM): realizada com o auxílio de enxada. Quando as plantas invasoras atingiam a altura de $45 \mathrm{~cm}$, em média, foram realizadas cinco operações anuais. $\mathrm{Na}$ ocasião da amostragem, as espécies dominantes encontradas na área foram: Cenchrus echinatus L. (timbete, Poaceae); Euphorbia heterophylla L. (leiteira, Euphorbiaceae); e Digitaria horizontalis Willd. (capim-colchão, Poaceae); c) Herbicida de pósemergência (HPOS): glyphosate foi aplicado com o auxílio de uma bomba costal, na dose de $2,0 \mathrm{~L} \mathrm{ha}^{-1} \mathrm{de}$ produto comercial e $0,72 \mathrm{~kg} \mathrm{ha}^{-1}$ de ingrediente ativo, na formulação de concentrado solúvel, $360 \mathrm{~g} \mathrm{~L}^{-1}$, com volume de calda de $400 \mathrm{~L} \mathrm{ha}^{-1}$. Na ocasião da amostragem, as espécies dominantes encontradas na área foram: Amaranthus viridis (caruru-de-mancha,
Amaranthaceae) e Commelina benghalensis L. (trapoeraba, Commelinaceae); d) Roçadora (ROÇA): o equipamento utilizado foi uma roçadora da marca Kamaq ${ }^{\circledR}$ modelo KD 132, com largura de corte de $1,32 \mathrm{~m}$. Na ocasião da amostragem, as espécies dominantes encontradas na área foram: Cyperus rotundus L. (tiririca, Cyperaceae); Cynodon dactylon (L.) Pers. (grama-seda, Poaceae); Amaranthus viridis (caruru-de-mancha, Amaranthaceae); e Brachiaria decumbens Stapf. (braquiária, Poaceae); e) Enxada rotativa (ENRT): o eixo da enxada rotativa tem cinco flanges, sendo as duas laterais com três facas e as três centrais, com seis facas cada uma. Sua profundidade de trabalho foi de, aproximadamente, $15 \mathrm{~cm}$. Na ocasião da amostragem, as espécies dominantes encontradas na área foram: Cyperus rotundus L. (tiririca, Cyperaceae); Cynodon dactylon (L.) Pers. (grama-seda, Poaceae); e Bidens pilosa L. (picão-preto, Compositae); f) Grade (GRAD): o equipamento é composto de duas seções dispostas em tandem e cada seção é equipada com sete discos lisos, com largura de corte de 1,3 m. Sua profundidade de trabalho foi de, aproximadamente, $10 \mathrm{~cm}$. Na ocasião da amostragem, as espécies dominantes encontradas na área foram: Cyperus rotundus L. (tiririca, Cyperaceae); Cynodon dactylon (L.) Pers. (gramaseda, Poaceae); e Brachiaria plantaginea (Link) Hitchc. (marmelada, Poaceae); e g) Herbicida de préemergência (HPRE): ingrediente ativo oxyfluorfen (2cloro-a,a,a-trifluoro-p-tolyl-3-ethoxy-4-nitrophenyl ether), na dose de $2,0 \mathrm{~kg} \mathrm{ha}^{-1}$ de produto comercial e $0,48 \mathrm{~kg} \mathrm{ha}^{-1} \mathrm{de}$ ingrediente ativo, na formulação de concentrado emulsionável, $240 \mathrm{~g} \mathrm{~L}^{-1}$ (Rodrigues \& Almeida, 2005), aplicado a um volume de calda de $400 \mathrm{~L} \mathrm{ha}^{-1}$. Para esta aplicação, cuidou-se para que o solo estivesse livre de restos culturais e plantas invasoras.

Os manejos escolhidos apresentaram características contrastantes, variando desde métodos manuais até capinas químicas e mecânicas. Desde a instalação do experimento no ano de 1977, os sistemas de manejo das plantas invasoras supracitados, no centro da entrelinha, foram os mesmos; o número médio de operações adotadas para o controle satisfatório das plantas daninhas, durante os anos, variou de acordo com o sistema de manejo adotado. Nas condições de manejo em que se utilizaram CAPM, HPÓS, ENRT e GRAD, foram realizadas oito operações no período de janeiro de 2006 a dezembro de 2007; no manejo ROÇA, realizaram-se nove operações no período; e para a aplicação do HPRE, foram seis aplicações no período - mais detalhes sobre a condução da área experimental podem ser obtidos em Alcântara \& Ferreira (2000a, b) e Alcântara et al. (2009).

Em dezembro de 2007, amostras indeformadas foram coletadas em anéis volumétricos de $2,54 \mathrm{~cm}$ de altura por $6,35 \mathrm{~cm}$ de diâmetro, no centro das entrelinhas dos cafeeiros (2,0 m do caule) e em uma mata nativa adjacente à área de estudo, nas 
profundidades de 0-3, 10-13 e 25-28 cm. Essas camadas foram utilizadas pelo fato de a maior influência do manejo de plantas daninhas ocorrer até $15 \mathrm{~cm}$ de profundidade (Alcântara \& Ferreira, 2000a), provavelmente porque o sistema radicular das plantas invasoras se concentra nesta camada.

As determinações químicas e físicas do LVdf (óxidos totais, granulometria, $\mathrm{pH}$ em água, $\mathrm{Ca}, \mathrm{Mg}, \mathrm{Al}, \mathrm{K}$ trocáveis, CTC efetiva, CTC a pH 7,0 e C orgânico total) foram feitas no solo excedente (partes superior e inferior) dos anéis volumétricos. $\mathrm{O}$ método de amostragem utilizado neste estudo foi similar ao utilizado por Potter \& Chichester (1993), quando esses autores avaliaram propriedades químicas e físicas de um solo sob diferentes preparos.

$\mathrm{O}$ valor de $\mathrm{pH}$ foi determinado em água, na relação de 1:2,5 $\left(10 \mathrm{~cm}^{3}\right.$ de TFSA mais $25 \mathrm{~mL}$ de $\left.\mathrm{H}_{2} \mathrm{O}\right)$, e os teores de $\mathrm{Ca}, \mathrm{Mg}$ e $\mathrm{Al}$ trocáveis foram determinados após extração com $\mathrm{KCl}\left(1 \mathrm{~mol} \mathrm{~L}^{-1}\right)$, na relação de $10 \mathrm{~cm}^{3}$ de TFSA para $100 \mathrm{~mL}$ do extrator, com agitação durante 5 min e decantação durante $16 \mathrm{~h}$. As determinações de $\mathrm{Ca}$ e $\mathrm{Mg}$ foram feitas por espectrofotometria de absorção atômica, utilizando-se para isso soluções-padrão de Ca e Mg contendo solução de $\mathrm{La}$ e $\mathrm{KCl}$ nas mesmas concentrações dos extratos.

$\mathrm{O}$ teor de $\mathrm{K}$ foi determinado em espectrofotômetro de chama, após extração com a solução Mehlich-1 (HCl $\left.0,05 \mathrm{~mol} \mathrm{~L}^{-1}+\mathrm{H}_{2} \mathrm{SO}_{4} 0,0125 \mathrm{~mol} \mathrm{~L}^{-1}\right)$, na relação de $10 \mathrm{~cm}^{3}$ de TFSA para $100 \mathrm{~mL}$ do extrator, com agitação durante 5 min e decantação durante $16 \mathrm{~h}$.

A extração de $\mathrm{H}+\mathrm{Al}$ foi realizada com $\mathrm{Ca}(\mathrm{OAc})_{2}$ $0,5 \mathrm{~mol} \mathrm{~L}^{-1}, \mathrm{pH} 7$, na relação de $5 \mathrm{~cm}^{3}$ de TFSA para $75 \mathrm{~mL}$ do extrator, com $10 \mathrm{~min}$ de agitação e decantação por $16 \mathrm{~h}$.

A capacidade efetiva de troca de cátions (CTC efetiva ou CTC a $\mathrm{pH}$ natural) foi determinada pelo somatório de $\mathrm{Ca}+\mathrm{Mg}+\mathrm{K}+\mathrm{Al}$ trocáveis, e a capacidade de troca de cátions a pH 7 (CTC a pH 7,0), pelo somatório de $\mathrm{Ca}+\mathrm{Mg}+\mathrm{K}+(\mathrm{H}+\mathrm{Al})$.

Os teores de C orgânico do solo foram obtidos pelo método da combustão via úmida com oxidação do $\mathrm{C}$ orgânico com $5 \mathrm{~mL}$ de $\mathrm{K}_{2} \mathrm{Cr}_{2} \mathrm{O}_{7}$ (dicromato de potássio) 0,167 $\mathrm{mol} \mathrm{L}^{-1}$ e $10 \mathrm{~mL}$ de $\mathrm{H}_{2} \mathrm{SO}_{4}$ (ácido sulfúrico) concentrado (Walkley \& Black, 1934). As análises químicas do solo seguiram os métodos analíticos recomendados pela CFSEMG (1999).

Os resultados das análises de $\mathrm{pH}, \mathrm{Ca}, \mathrm{Mg}, \mathrm{Al}, \mathrm{K}$, C orgânico do solo, CTC a pH natural e CTC a pH 7,0 foram submetidos à análise de variância, e a comparação das médias foi feita pelo teste de ScottKnott, utilizando-se o programa estatístico Sisvar (Ferreira, 2000).

Para verificar a relação entre as variáveis teor de C orgânico do solo (COS) e capacidade de troca de cátions efetiva (CTC a pH natural) e a capacidade de troca de cátions a pH 7, regressões lineares com intervalos de confiança a $95 \%$ foram obtidas.

\section{RESULTADOS E DISCUSSÃO}

Os valores de $\mathrm{pH}$ do solo sob mata nativa nas três profundidades estudadas foram inferiores aos obtidos no solo sob lavoura cafeeira (Quadro 1). Neste solo, os valores de $\mathrm{pH}$ não foram alterados pelos manejos de plantas invasoras na profundidade de $0-3 \mathrm{~cm}$. No entanto, com o aumento da profundidade, o valor de $\mathrm{pH}$ do solo manejado com HPRE reduziu em relação aos demais sistemas de manejo das plantas invasoras (Quadro 1), igualando-se ao valor de $\mathrm{pH}$ do solo sob mata nativa na profundidade de $25-28 \mathrm{~cm}$. A ausência de plantas invasoras nas entrelinhas dos cafeeiros proporcionada pelo manejo HPRE pode ter favorecido a acidificação do solo em subsuperfície, provavelmente devido à mobilização de ácidos, orgânicos e inorgânicos, da superfície.

Na profundidade de 10-13 cm, os maiores valores de $\mathrm{pH}$ foram observados para o solo sob os manejos SCAP, CAPM e GRAD. Esses manejos, na ocasião da amostragem (dezembro de 2007), apresentavam maior produção de matéria vegetal, como foi observado no campo, inclusive no manejo GRAD, com predominância da espécie tiririca.

Pelos dados apresentados no quadro 1, observa-se que o Ca foi o cátion dominante no complexo sortivo, ocupando entre 65 e $80 \%$ da CTC efetiva no solo cultivado com cafeeiros e $56 \%$ no solo sob mata nativa. Esse resultado pode ser devido à maior seletividade de troca do Ca em relação ao Mg e K (Loyola Junior \& Pavan, 1989). Além disso, a maior proporção de Ca na CTC efetiva no solo sob lavoura cafeeira, em relação ao LVdf sob mata nativa, pode estar associada à maior ciclagem desse nutriente promovida pela biomassa das plantas invasoras, que apresentam quantidades consideráveis de Ca (Meda et al., 2002), uma vez que a aplicação de corretivos (calcário) e fertilizantes foi feita desde a implantação da lavoura sobre a projeção dos cafeeiros.

Meda et al. (2002) observaram que a presença de plantas invasoras na superfície do solo pode aumentar a eficiência da calagem na correção da acidez da subsuperfície do solo, em virtude da contribuição da composição química das plantas invasoras e dos extratos vegetais, que apresentam quantidades consideráveis de Ca, Mg e K (Meda et al., 2002). Além dos efeitos químicos para a correção em subsuperfície, espera-se ainda o efeito físico dos bioporos (canais formados pelas raízes, minhocas e insetos de solo) no transporte de partículas finas dos corretivos da superfície para a subsuperfície, devido ao movimento descendente de água no solo, proporcionando a solubilização do $\mathrm{CaCO}_{3}$ em maiores profundidades (Sá, 1993). Portanto, a utilização constante de herbicidas de pré-emergência - que favorece o encrostamento superficial resultante do impacto direto das gotas de chuva - provavelmente impede a correção em subsuperfície, por proporcionar ausência de plantas invasoras e menor bioporosidade na superfície. 
Quadro 1. Atributos químicos de um Latossolo sob lavoura cafeeira submetido a diferentes manejos das plantas invasoras, em três profundidades

\begin{tabular}{|c|c|c|c|c|c|}
\hline \multirow{2}{*}{ Manejo de plantas invasoras } & \multirow{2}{*}{$\mathrm{pH} \mathrm{H}_{2} \mathrm{O}$} & \multicolumn{4}{|c|}{ Cátions trocáveis } \\
\hline & & $\mathbf{C a}$ & Mg & $\mathrm{Al}$ & $\mathbf{K}$ \\
\hline & & \multicolumn{4}{|c|}{$\mathrm{cmol}_{\mathrm{c}} \mathrm{dm}^{-3}$} \\
\hline & & \multicolumn{4}{|c|}{ Profundidade $0-3 \mathrm{~cm}$} \\
\hline Mata nativa & $5,3 \mathrm{~B}$ & $1,8 \mathrm{D}$ & $1,0 \mathrm{C}$ & $0,1 \mathrm{~A}$ & $0,3 \mathrm{C}$ \\
\hline Sem capina & $6,0 \mathrm{~A}$ & $7,8 \mathrm{~A}$ & $2,8 \mathrm{~A}$ & $0,1 \mathrm{~A}$ & $0,6 \mathrm{~A}$ \\
\hline Capina manual & $6,3 \mathrm{~A}$ & $5,3 \mathrm{~B}$ & $1,3 \mathrm{~B}$ & $0,1 \mathrm{~A}$ & $0,4 \mathrm{~B}$ \\
\hline Herbicida de pós-emergência & $6,0 \mathrm{~A}$ & $5,2 \mathrm{~B}$ & $1,4 \mathrm{~B}$ & $0,1 \mathrm{~A}$ & $0,5 \mathrm{~A}$ \\
\hline Roçadora & $5,9 \mathrm{~A}$ & $5,4 \mathrm{~B}$ & $1,5 \mathrm{~B}$ & $0,0 \mathrm{~A}$ & $0,5 \mathrm{~A}$ \\
\hline Enxada rotativa & $5,8 \mathrm{~A}$ & $4,7 \mathrm{~B}$ & $1,2 \mathrm{~B}$ & $0,1 \mathrm{~A}$ & $0,5 \mathrm{~A}$ \\
\hline Grade & $6,0 \mathrm{~A}$ & $4,5 \mathrm{~B}$ & $1,2 \mathrm{~B}$ & $0,1 \mathrm{~A}$ & $0,6 \mathrm{~A}$ \\
\hline Herbicida de pré-emergência & $6,0 \mathrm{~A}$ & $3,7 \mathrm{C}$ & $0,7 \mathrm{C}$ & $0,1 \mathrm{~A}$ & $0,2 \mathrm{C}$ \\
\hline & & \multicolumn{4}{|c|}{ Profundidade $10-13 \mathrm{~cm}$} \\
\hline Mata nativa & $5,1 \mathrm{D}$ & $0,6 \mathrm{C}$ & $0,3 \mathrm{C}$ & $0,2 \mathrm{~A}$ & $0,2 \mathrm{~B}$ \\
\hline Sem capina & $7,0 \mathrm{~A}$ & $5,3 \mathrm{~A}$ & $1,3 \mathrm{~A}$ & $0,1 \mathrm{~A}$ & $0,2 \mathrm{~B}$ \\
\hline Capina manual & $7,0 \mathrm{~A}$ & $3,2 \mathrm{~B}$ & $0,8 \mathrm{~B}$ & $0,1 \mathrm{~A}$ & $0,2 \mathrm{~B}$ \\
\hline Herbicida de pós-emergência & $6,7 \mathrm{~B}$ & $3,2 \mathrm{~B}$ & $0,7 \mathrm{~B}$ & $0,1 \mathrm{~A}$ & $0,3 \mathrm{~A}$ \\
\hline Roçadora & $6,4 \mathrm{~B}$ & $3,3 \mathrm{~B}$ & $0,7 \mathrm{~B}$ & $0,0 \mathrm{~A}$ & $0,2 \mathrm{~B}$ \\
\hline Enxada rotativa & $6,4 \mathrm{~B}$ & $3,0 \mathrm{~B}$ & $0,7 \mathrm{~B}$ & $0,1 \mathrm{~A}$ & $0,3 \mathrm{~A}$ \\
\hline Grade & $7,0 \mathrm{~A}$ & $2,8 \mathrm{~B}$ & $0,7 \mathrm{~B}$ & $0,1 \mathrm{~A}$ & $0,3 \mathrm{~A}$ \\
\hline Herbicida de pré-emergência & $5,7 \mathrm{C}$ & $1,3 \mathrm{C}$ & $0,3 \mathrm{C}$ & $0,1 \mathrm{~A}$ & $0,1 \mathrm{~B}$ \\
\hline \multicolumn{6}{|c|}{ Profundidade $25-28 \mathrm{~cm}$} \\
\hline Mata nativa & $5,2 \mathrm{C}$ & $0,2 \mathrm{C}$ & $0,1 \mathrm{~B}$ & $0,3 \mathrm{~A}$ & $0,1 \mathrm{~B}$ \\
\hline Sem capina & $7,0 \mathrm{~A}$ & $3,6 \mathrm{~A}$ & $0,9 \mathrm{~A}$ & $0,1 \mathrm{~A}$ & $0,1 \mathrm{~B}$ \\
\hline Capina manual & $6,7 \mathrm{~A}$ & $1,9 \mathrm{~B}$ & $0,6 \mathrm{~A}$ & $0,1 \mathrm{~A}$ & $0,1 \mathrm{~B}$ \\
\hline Herbicida de pós-emergência & $6,1 \mathrm{~B}$ & $1,6 \mathrm{~B}$ & $0,4 \mathrm{~A}$ & $0,1 \mathrm{~A}$ & $0,1 \mathrm{~B}$ \\
\hline Roçadora & $6,6 \mathrm{~A}$ & $2,0 \mathrm{~B}$ & $0,4 \mathrm{~A}$ & $0,0 \mathrm{~A}$ & $0,2 \mathrm{~B}$ \\
\hline Enxada rotativa & $6,6 \mathrm{~A}$ & $1,9 \mathrm{~B}$ & $0,5 \mathrm{~A}$ & $0,1 \mathrm{~A}$ & $0,1 \mathrm{~B}$ \\
\hline Grade & $6,7 \mathrm{~A}$ & $2,3 \mathrm{~B}$ & $0,6 \mathrm{~A}$ & $0,1 \mathrm{~A}$ & $0,3 \mathrm{~A}$ \\
\hline Herbicida de pré-emergência & $5,0 \mathrm{C}$ & $0,8 \mathrm{C}$ & $0,2 \mathrm{~B}$ & $0,1 \mathrm{~A}$ & $0,1 \mathrm{~B}$ \\
\hline
\end{tabular}

Médias seguidas da mesma letra maiúscula nas colunas, dentro de cada atributo e profundidade, não diferem entre si a $5 \%$ pelo teste de Scott-Knott.

O manejo SCAP aumentou o teor de Ca no solo nas três profundidades estudadas e de $\mathrm{Mg}$ nas profundidade de $0-3$ e $10-13 \mathrm{~cm}$ em relação aos demais sistemas de manejo de plantas invasoras e ao solo sob mata nativa (Quadro 1), o que pode ser atribuído à maior deposição de resíduos das plantas invasoras, favorecendo a disponibilidade desses cátions - como ressaltado anteriormente. Além disso, as raízes das plantas invasoras podem retirar esses nutrientes em camadas profundas e liberá-los na superfície, dependendo das plantas invasoras.

O teor de Ca verificado no solo sob o manejo HPRE na profundidade de 10-13 cm foi igual ao do solo sob mata nativa e inferior ao teor desse nutriente no solo sob lavoura cafeeira, em relação aos demais sistemas de manejo.

Pavan et al. (1985) constataram que a cobertura vegetal na superfície do solo proporcionada pela ceifa das plantas invasoras em lavouras cafeeiras promoveu aumento nos teores de $\mathrm{Ca}, \mathrm{Mg}, \mathrm{Ke} \mathrm{C}$ orgânico do solo, em comparação com os manejos enxada manual, enxada rotativa, herbicida e adubação verde. Resultados semelhantes foram obtidos por Alcântara et al. (2009) quando estes autores avaliaram os atributos químicos do LVdf no período de 1980 a 1995.

Os teores de $\mathrm{Al}$ trocável não foram alterados pelos diferentes manejos de plantas invasoras nas três profundidades estudadas (Quadro 1), provavelmente em razão do baixo teor natural desse elemento no solo, sob mata nativa adjacente à área de estudo, os teores de $\mathrm{Al}$ no solo foram de $0,1,0,2$ e $0,3 \mathrm{cmol}_{\mathrm{c}} \mathrm{dm}^{-3}$, nas profundidades de 0-3, 10-13 e 25-28 cm, respectivamente. Além disso, deve-se ressaltar que, mesmo para valores de $\mathrm{pH}$ em água acima de 6,0, observa-se Al trocável no solo sob diferentes manejos de plantas invasoras (Quadro 1). Para solos do Estado de Minas Gerais, Vasconcelos et al. (1994) demonstraram que o $\mathrm{pH}$ não explica adequadamente a variação do $\mathrm{Al}$ em todos os solos. Esses autores ressaltaram que o extrator $\mathrm{KCl} 1 \mathrm{~mol} \mathrm{~L}^{-1}$ extrai tanto $\mathrm{Al}$ como hidrogênio. Assim, pode haver influência do teor de $\mathrm{H}$ proveniente da matéria orgânica do solo e, dependendo do teor de matéria orgânica, o teor desse cátion pode ser maior ou menor.

Os teores de $\mathrm{K}$ foram alterados pelos diferentes sistemas de manejo de plantas invasoras nas três 
profundidades estudadas (Quadro 1), o que pode ser atribuído à abundância desse cátion no tecido vegetal na forma de $\mathrm{K}^{+}$. A decomposição dos restos vegetais libera-o na sua totalidade rapidamente; com isso, estão propensos a perdas por lixiviação (Borkert et al., 2003). $\mathrm{O} \mathrm{K}$ e o $\mathrm{N}$ foram os nutrientes de maior quantidade na biomassa de oito espécies de plantas invasoras (Meda et al., 2002) e de cinco plantas de cobertura utilizadas em sistema de semeadura direta (Borkert et al., 2003).

Os manejos SCAP, HPOS, ROÇA, ENRT e GRAD aumentaram os valores de $\mathrm{K}$ trocável na profundidade de 0-3 cm em relação aos manejos CAPM e HPRE e ao solo sob MATA (Quadro 1), indicando que as plantas invasoras podem estar servindo para reciclar o K, depositando-o na camada superficial, no centro das entrelinhas dos cafeeiros. Tanto o solo sob MATA quanto o sob manejo HPRE reduziram o teor de K trocável em relação ao solo sob CAPM. Observações de campo mostraram que o solo sob manejo HPRE apresenta maior erosão laminar seguido pelo manejo CAPM, indicando que o teor de K trocável na camada superficial pode estar relacionado à erosão do solo. Perdas de K trocável em sedimentos do solo em uma lavoura cafeeira foram observadas por Carvalho et al. (2007) sob diferentes sistemas de manejo do cafeeiro no sul de Minas Gerais. Esses autores ressaltaram que o K apresenta menor retenção pelos constituintes do solo, quando comparado ao $\mathrm{Ca}$ e ao $\mathrm{Mg}$, o que facilita o transporte pela água da enxurrada.

Os teores de $\mathrm{K}$ no solo aumentaram na profundidade de 10-13 cm nos manejos HPOS, ENRT e GRAD em relação ao solo sob MATA e aos demais sistemas de manejo de plantas invasoras (Quadro 1). As espécies de plantas invasoras sob os manejos HPOS, ENRT e GRAD podem ter contribuído para a movimentação do K trocável no perfil, aumentando os teores na profundidade de $10-13 \mathrm{~cm}$.

No manejo GRAD, o teor de K no solo aumentou na profundidade de $25-28 \mathrm{~cm}$ em relação ao solo sob MATA e sob os demais manejos de plantas invasoras (Quadro 1), indicando que houve efeito do revolvimento do solo com esse equipamento na movimentação do $\mathrm{K}$ trocável. Esses resultados concordam com os observados por Oliveira et al. (2004), os quais constataram que o revolvimento do solo com arado de disco aumenta o valor do K trocável nas profundidades de 10-20, 20-30 e 30-40 cm, em comparação com o solo sob Cerrado e sistema de semeadura direta.

Os diferentes manejos de plantas invasoras na lavoura cafeeira alteraram os valores da CTC efetiva, da CTC a pH 7 e dos teores de C orgânico do solo (Quadro 2), corroborando os resultados de Pavan et al. (1995), Ciotta et al. (2003) e Alcântara et al. (2009).

O manejo SCAP proporcionou ao LVdf os maiores valores de CTC a pH 7,0 nas três profundidades, mesmo quando comparado ao solo sob MATA (Quadro 2), evidenciando a importância do aporte de biomassa a partir das plantas invasoras na geração de cargas nesse solo. Além da quantidade de biomassa produzida sob este manejo, é importante salientar que a qualidade da matéria orgânica também pode estar influenciando a alteração dos atributos químicos.

A alteração da CTC a pH 7,0 em estudos de longa duração pode ser devido à alteração da força iônica da solução do solo e consequentemente da atividade de alguns cátions, o que promove a alteração desse atributo com o manejo. Em solos com predominância de minerais de atividade baixa, após 21 anos sob o sistema plantio direto, Ciotta et al. (2003) observaram aumento da CTC a pH 7,0 em relação ao solo sob o sistema de plantio convencional.

O manejo HPRE reduziu os valores de CTC efetiva nas três profundidades estudadas, em relação aos demais manejos de plantas invasoras utilizados na lavoura cafeeira (Quadro 2). Quando comparado ao solo sob MATA, o solo sob o manejo HPRE manteve os valores de CTC efetiva nas três profundidades. Os demais manejos de plantas invasoras proporcionaram valores intermediários de CTC efetiva em relação ao solo sob os manejos SCAP e MATA/HPRE, nas três profundidades (Quadro 2). A CTC efetiva é um atributo que varia simultaneamente em função do $\mathrm{pH}$ e da quantidade de matéria orgânica do solo. Portanto, o aumento do $\mathrm{pH}$ do solo é uma estratégia fundamental para o aumento do número de cargas negativas nos solos de regiões tropicais, uma vez que os solos destas regiões são compostos por minerais de baixa atividade (Pavan et al., 1985; Meurer et al., 2006).

Os manejos SCAP e HPRE proporcionaram, respectivamente, os maiores e menores valores de COS na profundidade de 0-3 cm. Os manejos ROÇA, ENRT e GRAD proporcionaram valores de COS menores que o manejo SCAP, porém superiores aos manejos CAPM e HPOS. O revolvimento do solo proporcionado pela enxada rotativa e grade e a cobertura vegetal constante proporcionada pela roçadora favorecem interações organominerais, que protegem a matéria orgânica parcialmente da fotodegradação e do ataque de microrganismos.

Os diferentes sistemas de manejo de plantas invasoras não alteraram os valores da CTC a pH 7 e COS na profundidade de 25-28 cm (Quadro 2), evidenciando que as maiores influências do manejo de plantas invasoras em atributos químicos ocorrem até a profundidade de $10-13 \mathrm{~cm}$, corroborando assim resultados de Alcântara \& Ferreira (2000a).

Pelas figura 1a-f, observa-se que, com o aumento do teor de $\mathrm{C}$ orgânico no solo, ocorre aumento linear nos valores de CTC efetiva e CTC a pH 7. Observamse relações entre o conteúdo de $\mathrm{C}$ orgânico total do solo e a CTC efetiva em 59, 60 e $47 \%$ dos casos e de 65,55 e $46 \%$ entre o conteúdo de $\mathrm{C}$ orgânico total do solo e a CTC a pH 7,0 para as profundidades $0-3$, 10-13 e 25-28 cm (Figuras 1a,f). 
Quadro 2. Capacidade efetiva de troca de cátions (CTC a pH natural), CTC a pH 7 e teor de carbono orgânico de um Latossolo sob lavoura cafeeira submetido a diferentes manejos de plantas invasoras, em três profundidades

\begin{tabular}{|c|c|c|c|}
\hline Manejo de plantas invasoras & CTC efetiva & CTC a pH 7 & $\cos$ \\
\hline & \multicolumn{2}{|c|}{$\longrightarrow \mathrm{cmol}_{\mathrm{c}} \mathrm{dm}^{-3} \longrightarrow$} & $\mathrm{g} \mathrm{kg}^{-1}$ \\
\hline & & Profundidade $0-3 \mathrm{~cm}$ & \\
\hline Mata nativa & $3,2 \mathrm{D}$ & $7,1 \mathrm{D}$ & $15,7 \mathrm{C}$ \\
\hline Sem capina & $11,3 \mathrm{~A}$ & $13,5 \mathrm{~A}$ & $24,5 \mathrm{~A}$ \\
\hline Capina manual & $7,1 \mathrm{~B}$ & $9,6 \mathrm{C}$ & $15,1 \mathrm{C}$ \\
\hline Herbicida de pós-emergência & $7,2 \mathrm{~B}$ & $10,8 \mathrm{~B}$ & $16,8 \mathrm{C}$ \\
\hline Roçadora & $7,4 \mathrm{~B}$ & $10,8 \mathrm{~B}$ & $21,2 \mathrm{~B}$ \\
\hline Enxada rotativa & $6,6 \mathrm{~B}$ & $10,2 \mathrm{~B}$ & $20,3 \mathrm{~B}$ \\
\hline Grade & $6,4 \mathrm{~B}$ & $9,3 \mathrm{C}$ & $18,6 \mathrm{~B}$ \\
\hline \multirow[t]{2}{*}{ Herbicida de pré-emergência } & $4,7 \mathrm{C}$ & $8,5 \mathrm{C}$ & $12,0 \mathrm{D}$ \\
\hline & & Profundidade $10-13 \mathrm{~cm}$ & \\
\hline Mata nativa & $1,3 \mathrm{C}$ & $6,1 \mathrm{~B}$ & $12,8 \mathrm{~A}$ \\
\hline Sem capina & $6,8 \mathrm{~A}$ & $8,6 \mathrm{~A}$ & $14,3 \mathrm{~A}$ \\
\hline Capina manual & $4,4 \mathrm{~B}$ & $6,4 \mathrm{~B}$ & $11,0 \mathrm{~B}$ \\
\hline Herbicida de pós-emergência & $4,4 \mathrm{~B}$ & $6,9 \mathrm{~B}$ & $11,4 \mathrm{~B}$ \\
\hline Roçadora & $4,2 \mathrm{~B}$ & $6,7 \mathrm{~B}$ & $12,8 \mathrm{~A}$ \\
\hline Enxada rotativa & $4,2 \mathrm{~B}$ & $6,7 \mathrm{~B}$ & $10,1 \mathrm{~B}$ \\
\hline Grade & $3,9 \mathrm{~B}$ & $6,1 \mathrm{~B}$ & $10,1 \mathrm{~B}$ \\
\hline \multirow[t]{2}{*}{ Herbicida de pré-emergência } & $2,0 \mathrm{C}$ & $5,9 \mathrm{~B}$ & $9,3 \mathrm{~B}$ \\
\hline & & Profundidade $25-28 \mathrm{~cm}$ & \\
\hline Mata nativa & $0,7 \mathrm{D}$ & $5,4 \mathrm{~B}$ & $10,4 \mathrm{~A}$ \\
\hline Sem capina & $4,7 \mathrm{~A}$ & $6,5 \mathrm{~A}$ & $9,9 \mathrm{~A}$ \\
\hline Capina manual & $2,7 \mathrm{C}$ & $4,8 \mathrm{~B}$ & $8,3 \mathrm{~A}$ \\
\hline Herbicida de pós-emergência & $2,2 \mathrm{C}$ & $4,8 \mathrm{~B}$ & $8,3 \mathrm{~A}$ \\
\hline Roçadora & $2,5 \mathrm{C}$ & $4,8 \mathrm{~B}$ & $8,7 \mathrm{~A}$ \\
\hline Enxada rotativa & $2,6 \mathrm{C}$ & $4,7 \mathrm{~B}$ & $8,5 \mathrm{~A}$ \\
\hline Grade & $3,3 \mathrm{~B}$ & $5,5 \mathrm{~B}$ & $9,5 \mathrm{~A}$ \\
\hline Herbicida de pré-emergência & $1,1 \mathrm{D}$ & $4,8 \mathrm{~B}$ & $7,4 \mathrm{~A}$ \\
\hline
\end{tabular}

Médias seguidas da mesma letra nas colunas, dentro de cada atributo, não diferem entre si a $5 \%$ pelo teste de Scott-Knott.

Para Latossolos do Estado de São Paulo com teores de argila semelhantes aos utilizados neste estudo, Raij (1969) observou que a matéria orgânica pode ter contribuição relativa de até $90 \%$ para a CTC total do solo. Para um Latossolo do Estado do Paraná submetido a cinco manejos das plantas daninhas na lavoura cafeeira, a contribuição relativa da CTC da matéria orgânica para a CTC a pH 7 foi de 56 a $62 \%$ e de 77 a 81 \% para a CTC efetiva; a matéria orgânica apresentou a maior contribuição para as cargas variáveis do solo (Pavan et al., 1995). No presente estudo, há menor relação entre o teor de COS e a CTC efetiva na profundidade de $0-3 \mathrm{~cm}$, em relação à CTC a pH 7 (Figura 1a). Esse resultado pode ser atribuído à influência do $\mathrm{pH}$ do solo para a CTC efetiva, reduzindo a contribuição do $\mathrm{C}$ orgânico.

O aumento da CTC do solo cultivado com cafeeiros e submetido a diferentes sistemas de manejo de plantas invasoras promove aumento das cargas negativas, já que a CTC pode ser entendida como uma estimativa indireta das cargas negativas do solo. Isso se deve aos grupos funcionais que compõem a matéria orgânica do solo, os quais determinam que a capacidade desses constituintes do solo de perder ou receber íons $\mathrm{H}^{+}$ (dissociação ou protonação) é a responsável pela geração de cargas da matéria orgânica do solo. A protonação da matéria orgânica do solo ocorre somente em pH inferior a 3,0 (PCZ da matéria orgânica em torno de 3,0), uma vez que ácidos fúlvicos e húmicos comportam-se como polímeros descarregados, ao passo que em valores de $\mathrm{pH}>3,0$ esses ácidos se comportam como polieletrólitos carregados negativamente; assim, em $\mathrm{pH}$ dos solos agrícolas ocorre a dissociação de grupos carboxílicos, gerando cargas negativas no solo (Silva et al., 2006).

\section{CONCLUSÕES}

1. A manutenção das plantas invasoras nas entrelinhas dos cafeeiros, adotada no manejo sem capina, contribuiu positivamente para as alterações dos atributos químicos (Ca trocável, CTC efetiva e CTC a pH 7,0) nas três profundidades estudadas. Além disso, elevou o teor de C orgânico total na profundidade de $0-3 \mathrm{~cm}$ e pode contribuir para o aumento e a manutenção dos estoques de $\mathrm{C}$ em lavouras cafeeiras. 

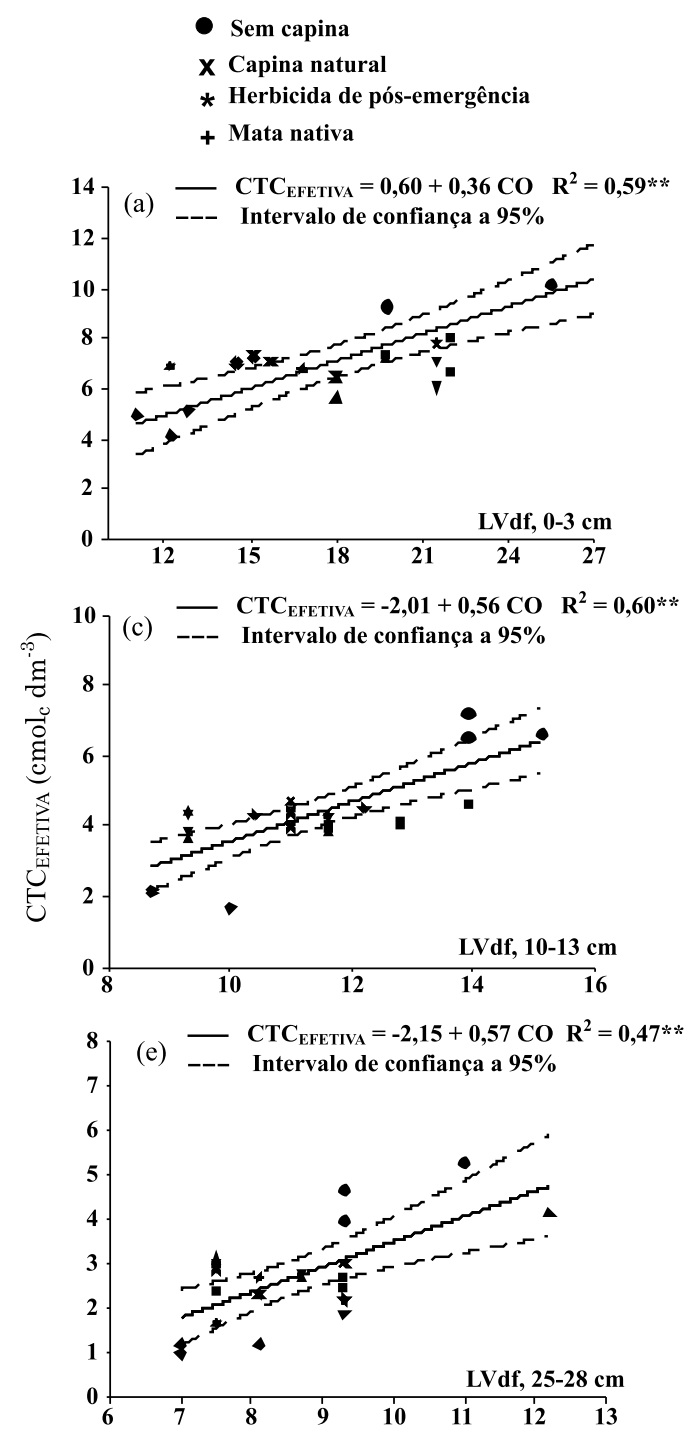

- Roçadora

$\nabla$ Enxada rotativa

Grade

- Herbicida de pré-emergência
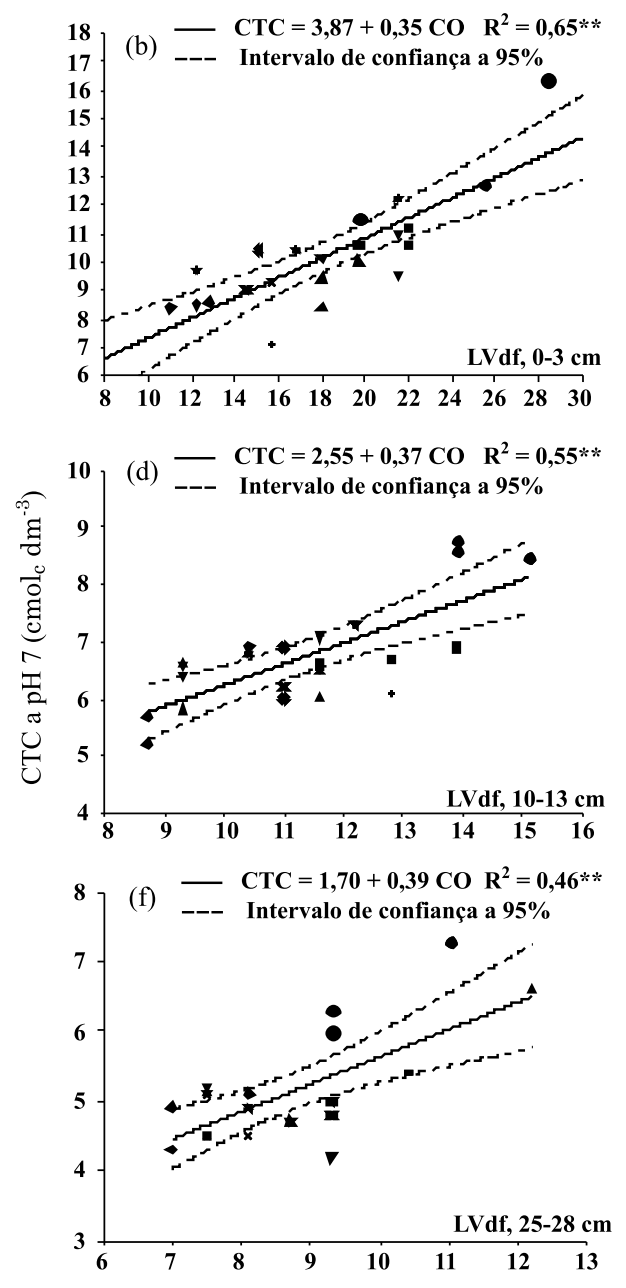

Carbono orgânico $\left(\mathrm{g} \mathrm{kg}^{-1}\right)$

Figura 1. Capacidade de troca de cátions efetiva (a, c, e) e CTC a pH 7 (b, d, f) em função do conteúdo de carbono orgânico de um Latossolo nas profundidades de 0-3, 10-13 e 25-28 cm, submetido a sistemas de manejo de plantas invasoras em uma lavoura cafeeira.

2. O manejo constante e por longo prazo (30 anos) com herbicida de pré-emergência reduziu os valores de $\mathrm{pH}$ nas profundidades de $10-13$ e $25-28 \mathrm{~cm}$ e do $\mathrm{Ca}$ trocável, Mg trocável e CTC efetiva nas três profundidades estudaadas, em relação aos demais manejos de plantas invasoras.

3. Os valores de CTC efetiva do Latossolo Vermelho distroférrico apresentaram relação com o teor de C orgânico total em 59,60 e $47 \%$ dos casos e para a CTC a pH 7,0 em 65, 55 e $46 \%$, para as profundidades $0-3,10-13$ e $25-28 \mathrm{~cm}$.

\section{AGRADECIMENTOS}

Os autores agradecem ao Consórcio Brasileiro de Pesquisa e Desenvolvimento do Café - CBP\&D/Café, pelo apoio financeiro para o desenvolvimento do presente estudo. Aos funcionários da Fazenda Experimental da EPAMIG em São Sebastião do Paraíso - MG, especialmente Paulo Guido de Azevedo, Homero José Lemos, Antônio Eguimar Pereira Xavier e Juracy Júnior de Oliveira, os quais contribuíram para a realização deste trabalho. $\mathrm{O}$ primeiro autor agradece à CAPES, pela concessão da bolsa de doutorado, e ao Dr. Marcos Antônio Pavan (ASO/ IAPAR), pela revisão e contribuição no manuscrito.

\section{LITERATURA CITADA}

AGBENIN, J.O. \& RAIJ, B.van. Rate process of calcium, magnesium and potassium desorption from variablecharge soils by mixed ion exchange resins. Geoderma, 93:141-157, 1999. 
ALCÂNTARA, E.N. \& FERREIRA, M.M. Efeito de métodos de controle de plantas daninhas na produção de café durante 30 anos. In: SIMPÓSIO DE PESQUISA DOS CAFÉS DO BRASIL. 6., Brasília, 2009. Anais... Brasília, Embrapa, 2009. CD ROM.

ALCÂNTARA, E.N. \& FERREIRA, M.M. Efeito de diferentes métodos de controle de plantas daninhas sobre a produção de cafeeiros instalados em Latossolo Roxo distrófico. Ci. Agrotec., 24:54-61, 2000a.

ALCÂNTARA, E.N. \& FERREIRA, M.M. Efeito de métodos de controle de plantas daninhas na cultura do cafeeiro (Coffea arabica L.) sobre a qualidade física do solo. R. Bras. Ci. Solo, 24:711-721, 2000b.

ALCÂNTARA, E.N.; NÓBREGA, J.C.A. \& FERREIRA, M.M. Métodos de controle de plantas daninhas no cafeeiro afetam os atributos químicos do solo. Ci. Rural, 39:749$757,2009$.

BAYER, C. \& MIELNICZUK, J. Características químicas do solo afetadas por métodos de preparo e sistemas de cultura. R. Bras. Ci. Solo, 21:105-112, 1997.

BAYER, C. \& MIELNICZUK, J. Dinâmica e função da matéria orgânica. In: SANTOS, G.A.; SILVA, L.S.; CANELLAS, L.P. \& CAMARGO, F.A.O., eds. Fundamentos da matéria orgânica do solo: Eecossistemas tropicais e subbtropicais. 2.ed. Porto Alegre, Metrópole, 2008. p.7-18.

BORKERT, C.M.; GAUDÊNCIO, C.A.; PEREIRA, J.E.; PEREIRA, L.R. \& OLIVEIRA JUNIOR, A. Nutrientes minerais na biomassa da parte aérea em culturas de cobertura do solo. Pesq. Agropec. Bras., 38:143-153, 2003.

CARVALHO, R.; SILVA, M.L.N.; AVANZI, J.C.; CURI, N. \& SOUZA, F.S. Erosão hídrica em Latossolo Vermelho sob diversos sistemas de manejo do cafeeiro no sul de Minas Gerais. Ci. Agrotec., 31:1679-1687, 2007.

CIOTTA, M.N.; BAYER, C.; FONTOURA, S.M.V.; ERNANI, P.R. \& ALBUQUERQUE, J.A. Matéria orgânica e aumento da capacidade de troca de cátions em solo com argila de atividade baixa sob plantio direto. Ci. Rural, 33:1161-1164, 2003.

COMISSÃO DE FERTILIDADE DO SOLO DO ESTADO DE MINAS GERAIS - CFSEMG. Recomendações para o uso de corretivos e fertilizantes em Minas Gerais - $5^{\text {a }}$ Aproximação. Viçosa, MG, 1999. 359p.

DORAN, J.W. Soil health and global sustainability: translating science into practice. Agric. Ecost. Environ., 88:119-127, 2002.

EMPRESA BRASILEIRA DE PESQUISA AGROPECUÁRIA EMBRAPA. Centro Nacional de Pesquisas de Solos. Sistema brasileiro de classificação de solos. 2.ed. Rio de Janeiro, Embrapa Solos, 2006. 306p.

FERREIRA, D.F. Análises estatísticas por meio do SISVAR para Windows 4. 0. In: REUNIÃO ANUAL DA REGIÃO BRASILEIRA DA SOCIEDADE INTERNACIONAL DE BIOMETRIA, 45., São Carlos 2000. Anais... São Carlos, UFSCAR, 2000. p.255-258.
FONTES, M.P.F.; CAMARGO, O.A.; \& SPOSITO, G. Eletroquímica das partículas coloidais e sua relação com a mineralogia de solos altamente intemperizados. Sci. Agric., 58:627-646, 2001.

LAL, R. World soils and global issues. Soil Tillage Res., 97:1-4, 2007.

LOYOLA JUNIOR, E. \& PAVAN, M.A. Seletividade de cátions em solos ácidos. R. Bras. Ci. Solo, 13:131-138, 1989.

MEDA, A.R.; PAVAN, M.A.; MIYAZAWA, M. \& CASSIOLATO, M.E. Plantas invasoras para melhorar a eficiência da calagem da correção da acidez subsuperficial do solo. R. Bras. Ci. Solo, 26:647-654, 2002.

MEUER, E.J.; RHENHEIMER, D. \& BISSANI, C.A. Fenômenos de sorção em solos. In: MEUER, E.J., ed. Fundamentos de química do solo. 3.ed. Porto Alegre, Evangraf, 2006. p.117-162.

OLIVEIRA, G.C.; DIAS JUNIOR, M.S.; RESCK, D.V.S. \& CURI, N. Caracterização química e físico-hídrica de um Latossolo Vermelho após vinte anos de manejo e cultivo do solo. R. Bras. Ci. Solo, 28:327-336, 2004.

PAVAN, M.A.; VIEIRA, M.J. \& ANDROCIOLI FILHO, A. Influência do manejo das plantas daninhas em lavoura cafeeira na capacidade de troca de cátions e cátions trocáveis em solo com cargas variáveis. Arq. Biol. Tecnol., 38:305-311, 1995.

PAVAN, M.A.; BINGHAM, F.T. \& PRATT, P.F. Chemical and mineralogical characteristics of selected acid soils of the State of Paraná, Brazil. Turrialba, 35:131-139, 1985.

PAYAN, F.; JONES, D.L. \& BEER, J. Dynamics of size-density fractions of soil organic matter following the addition of tree litter to organic coffee farms. Geoderma, 141:15-22, 2007.

POTTER, K.N. \& CHICHESTER, F.W. Physical and chemical properties of a Vertisol with continuous controlled-traffic, no-till management. Trans. ASAE, 36:95-99, 1993.

RAIJ, B.van. A capacidade de troca de cátions das frações orgânica e mineral em solos. Bragantia, 28:85-112, 1969.

RESENDE, M. \& SANTANA, D.P. Uso das relações Ki e Kr na estimativa da mineralogia para classificação de Latossolos. In: REUNIÃO DE CLASSIFICAÇÃO, CORRELAÇÃO DE SOLOS E INTERPRETAÇÃO DA APTIDÃO AGRÍCOLA, 3., Rio de Janeiro, 1988. Anais... Rio de Janeiro, Embrapa/ SNLCS/SBCS, 1988. p.225-232.

RODRIGUES, B.N. \& ALMEIDA, F.S. Guia de herbicidas. 5.ed. Londrina, 2005. 592p.

SÁ, J.C.M. Manejo da fertilidade do solo no sistema plantio direto. In: CNPT-EMBRAPA, FUNDACEP-FECOTRIGO, FUNDAÇÃO ABC. Plantio direto no Brasil. Passo Fundo, Aldeia Norte, 1993. p.37-60.

SILVA, L.S.; CAMARGO, F.A.O. \& CERETTA, C.A. Composição da fase sólida orgânica do solo. In: MEUER, E.J., ed. Fundamentos de química do solo. 3.ed. Porto Alegre, Evangraf, 2006. p.63-90. 
SPARKS, D.L. Inorganic soil components. In: SPARKS, D.L., ed. Environmental soil chemistry. San Diego, Academic Press, 1995. p.23-52.

VASCONCELLOS, C.A.; SANTANA, D.P. \& FERREIRA, L. Métodos de determinação da necessidade de calagem e características físico-químicas de alguns solos de Minas Gerais. Pesq. Agropec. Bras., 29: 1253-1263, 1994.
WALKLEY, A. \& BLACK, I.A. An examination of the Degtjareff method for determining soil organic matter and a proposed modification of the chromic acid titration method. Soil Sci., 37:29-38, 1934. 
